\title{
Scanning Various Nanomaterials and Variable Camouflages to Evaluate the Biocompatibility in Multi-organs of Embryos
}

\section{Bin Zheng}

Tianjin University

Mingming Guo

Tianjin University

Meijun Pang ( $\sim$ P20010@pku.edu.cn )

Tianjin University https://orcid.org/0000-0001-6553-0932

Dong Ming

Tianjin University

\section{Research}

Keywords: Nanomaterials, Multiple-organs toxicity, Quantum dots, Modification methods, Zebrafish

Posted Date: November 3rd, 2020

DOI: https://doi.org/10.21203/rs.3.rs-99384/v1

License: (c) (i) This work is licensed under a Creative Commons Attribution 4.0 International License. Read Full License 


\section{Abstract}

Background:

Nanomaterials are under a wide range of application prospects in human health and disease, such as medical imaging and drug delivery and treatment. With the gradual advent of the nano era, the safety of nanomaterials in biology must be evaluated more widely and deeply. Therefore, superficial safety assessment based on specific cell lines in vitro is difficult to meet the current needs. Multi-organs assessment in vivo will be beneficial in establishing a more comprehensive pathway for the understanding of nanomaterials-induced biotoxicity.

Results:

In this work, we employed a series of genetically modified zebrafish models for nanomaterials toxicity assessment. The results demonstrated that cadmium selenide (CdSe) quantum dot (QDs) was the most toxic after scanning some popular nanomaterials. Among modified methods of silica coating, core-shell structure development and organic molecular camouflage, the polyethylene glycol camouflage method exhibits a better performance of improving the biocompatibility of CdSe QDs in multiple-organs including hearts, nerves, blood vessels, and immune system.

\section{Conclusion:}

Our work provides an alternative paradigm for more in-depth and sensitive preclinical validation of biocompatibility, especially in neurotoxicity and cardiotoxicity of embryos, and a guidance for reducing the toxicity of biomedical materials.

\section{Introduction}

Due to the small size effect, surface effect, quantum size effect and macro quantum tunneling effect of nanomaterials, they present optical, acoustic, electrical, magnetic and temperature sensitive features that conventional materials do not.[1, 2] With the rapid development of many subjects, nanomaterials with excellent characteristics have a wide range of applications in disease treatment, medical imaging, biological detection, biosensing, etc.[3] Such as nanoenzymes in the elimination of ROS,[4] and gold nanoparticles (AuNPs) in biological detection,[5] the application of up-conversion nanoparticles (UCNPs) in imaging and tumor diagnosis,[6] quantum dots (QDs) as fluorescent markers widely used to image and track the migration of drugs in the body.[7, 8] The inevitable contact between humans and nanomaterials has made the safety of nanomaterials attract much attention all over the world.[9] The small size of nanoparticles makes it easier to enter cells or organelles through the gaps in the biological membrane, and combine with biological molecules or catalyze chemical reactions. The complex interaction could change the structure and the function of biological macromolecules and physique and ultimately lead to cellular cell and tissue toxicity. Some nanoparticles may trigger interactions with blood, affect major organs or accumulate in organs, and even cause immune responses. [10-12] 
In many current studies, the toxicity verification of nanomaterials is mainly based on the physiological activity test of specific cell lines in vitro, such as proliferation and apoptosis.[13, 14] Part of the evaluation involves body weight changes and macroscopic pathological changes of main organs, such as direct naked eye observation of main organs. $[15,16]$ Even when it comes to the pathological changes presented by Hematoxylin-eosin staining at the cellular level, it can only be appeared after a large amount of toxic substances are accumulated in the body. The sensitivity of toxicity detection and the comprehensive multiple organ toxicity evaluation are severely restricted by the above means, especially in neurotoxicity and cardiotoxicity. Therefore, more sensitive and systematic evaluation methods should be urgently employed to assess the biocompatibility of biomaterials in vivo. Zebrafish has been recognized as important model for human disease which has about 70\% similarity with humans.[17] Since zebrafish has regeneration capability in its organs such as fins, central nervous system (CNS), heart, pancreas, liver, and kidney, it has been used for different models of injury for example in cardiovascular, neurological, and metabolic diseases.[18-20] The technology of transgenic and genome editing is mature and efficient in zebrafish, which made zebrafish as a common model for genetic screen and disease models.[21-23] Moreover, the zebrafish embryos are transparent and develop externally, a variety of imaging modalities were applicable to live image of embryogenesis and disease occurrence.[24-26] With the help of fluorescence microscope and transgenic fluorescent reporters, researchers could specifically mark a variety of tissues such as vessels, neurons, inflammatory cells and examine the structural and functional phenotypes associated with genetic mutations and chemical exposure in vivo.[27] Hence, zebrafish embryos has been extensively applied for safety evaluation of medicines in preclinical studies because of its sensitivity to harmful substances and easy genetic modification.[28-30]

In this study, a variety of zebrafish embryos have been employed to scan the safety of popular nanomaterials. Among the widely used biomedical nanoparticles (AuNPs, UCNPs, C QDs, ZnO QDs and CdSe QDs), CdSe QDs indicated the most severe physiological toxicity, which caused cardiac dysfunction, vascular and neurons damage and triggered inflammation in zebrafish embryos. In order to reduce its toxicity and make it safer to use in organisms, CdSe QDs was camouflaged by wrapping with silicon dioxide $\left(\mathrm{SiO}_{2}\right)$, zinc sulfide ( $\left.\mathrm{ZnS}\right)$, polyethylene glycol (PEG). It is worth noting that PEG-wrapped CdSe QDs could significantly increase the survival rate, reduce the deformity rate. After the comprehensive evaluation of a series of transgenic zebrafish models obtained earlier.[31, 32] We were surprised to find that the toxicity of CdSe QDs to nerve, heart and blood vessel could be significantly reduced or even eliminated after PEG modification. Our approach might inspire future evaluation strategies of biocompatibility for biomedical nanoparticles before basic studies and clinical applications, especially in nerves, hearts and blood vessels of embryos that are particularly sensitive to harmful substances.

\section{Results And Discussion}

\subsection{Characterization and screening toxicity nanomaterials}


Gold nanoparticles, up-conversion nanoparticles, and quantum dots have a wide range of applications in imaging technology and treatment of disease, but it will have to be monitored more rigorously for toxic and side effects before they can actually be used for biomedical purposes. We prepared AuNPs, UCNPs, C QDs, ZnO QDs, and CdSe QDs to assess their toxic effects on zebrafish embryos. Through the analysis of dynamic light scattering (DLS), the size of AuNPs, UCNPs, C QDs, ZnO QDs and CdSe QDs are approximately $13 \mathrm{~nm}$ (Fig. 1A), $40 \mathrm{~nm}$ (Fig. 1B), $6 \mathrm{~nm}$ (Fig. 1C), $8 \mathrm{~nm}$ (Fig. 1D), $14 \mathrm{~nm}$ (Fig. 1E). The excitation wavelength of $C$ QDs is $405 \mathrm{~nm}$ and the emission wavelength is $570 \mathrm{~nm}$ (Figure S1). The excitation wavelength of $\mathrm{ZnO}$ QDs is $405 \mathrm{~nm}$, and the emission wavelength is $580 \mathrm{~nm}$ (Figure S2). The excitation wavelength of CdSe QDs is $405 \mathrm{~nm}$, and the emission wavelength is $600 \mathrm{~nm}$ (Figure S3). To explore their respective safety, the 4 hours post fertilization (4hpf) zebrafish embryos were incubated in hatching solution E3 with $0.2 \mathrm{mg} / \mathrm{mL}, 0.15 \mathrm{mg} / \mathrm{mL}, 0.1 \mathrm{mg} / \mathrm{mL}, 0.05 \mathrm{mg} / \mathrm{mL}$ nanomaterials respectively and analyzed by survival rate and hatching rate. The results showed all zebrafish in the $0.2 \mathrm{mg} / \mathrm{mL}$ and $0.15 \mathrm{mg} / \mathrm{mL} Z \mathrm{nO}$ QDs and CdSe QDs died, while the survival rates of the AuNPs, UCNPs, and C QDs group were about $80 \%-90 \%$ (Fig. 1F-I), which suggested that AuNPs, UCNPs, and C QDs are less toxic than ZnO QDs and CdSe QDs (Fig. 1F). When the zebrafish embryos were incubated in $0.1 \mathrm{mg} / \mathrm{mL}$, the survival rate and incubation rate of CdSe QDs was $60 \%$ which was the lowest among these nanomaterials (Fig. $1 \mathrm{~J}, \mathrm{~K}$ ). While the concentration was reduced to $0.05 \mathrm{mg} / \mathrm{mL}$, there was no significant difference in zebrafish survival rate and hatching rate after nanomaterials incubation (Fig. 1L, M). Taken together, our results demonstrated that CdSe QDs is the most toxic among these nanomaterials to zebrafish embryos and higher than $0.1 \mathrm{mg} / \mathrm{mL}$ will affect the zebrafish development including survival and hatching. Although some nanomaterials are highly toxic, how to improve their biocompatibility has become an important concern due to their unique properties and need to be used in biomedicine.

\subsection{Toxicity of CdSe QDs after different camouflage methods}

In order to decrease the toxicity of CdSe QDs and improve their application in biomedicine, we have selected commonly used modification methods and tested their toxicity reduction effects. We chose three modification methods and the principal was to wrap CdSe QDs with $\mathrm{SiO}_{2}, \mathrm{ZnS}$ and PEG. Because $\mathrm{SiO}_{2}$ has better biocompatibility, we believed that $\mathrm{SiO}_{2}$ wrapped CdSe QDs can decrease the toxicity of CdSe QDs (Fig. 2A). The second was a widespread modification method of CdSe QDs, which is to wrap ZnS around it to prepare CdSe@ZnS core-shell structure. According to previous literature reports, this method could reduce the toxicity of CdSe QDs (Fig. 2B). In the third type, almost non-toxic PEG was utilized to modify CdSe QDs (Fig. 2C). Since PEG modification was widely used in the modification of a variety of biomaterials to improve its biocompatibility and reduce toxicity. The particle size analysis and transmission electron microscopy (TEM) images showed that the success of these three modification methods (Fig. 2A-F). To evaluate different modification methods on zebrafish embryos toxicity, we first tested the different modifications on zebrafish survival rate, hatching rate and malformation rate (body bending) (Figure S4). Built on the previous results, we choose the concentration of $0.1 \mathrm{mg} / \mathrm{mL}$ for the next study. The zebrafish embryos were incubated in E3 solution with CdSe QDs, CdSe@SiO 2, CdSe@ZnS 
and CdSe@PEG and DMSO as control. Results indicated that CdSe@PEG group gets the highest survival rate was $100 \%$, hatching rate was $100 \%$ and low malformation rate was 0 compared with $\mathrm{CdSe} @ \mathrm{SiO}_{2}$ were 80\%, 70\%, 10\%, CdSe@ZnS group were 75\%, 70\%, 50\%. CdSe@SiO 2 , CdSe@ZnS group has similar survival rate, while CdSe@ZnS group has lower hatching rate was 70\% and higher malformation rate was $50 \%$ (Fig. 2G-I). These different modification methods analyses display that CdSe QDs with PEG modification significantly reduced its toxicity during zebrafish development. These results also suggest that PEG modification is a better way to improve the biocompatibility of nanoparticles, although the camouflage of silicon dioxide and core-shell structure encapsulation can reduce the toxicity of nanoparticles.

\subsection{Cardiotoxicity of CdSe QDs before and after PEG camouflage}

The heart is the main organ supplying blood to the whole body, so nanoparticles entering the body will be the first to enter the heart through the blood circulation. The embryonic heart is also the most active and sensitive period of life, so cardiotoxicity should be tested first. CdSe QDs has a certain effect on zebrafish cardiovascular development. We first detect the CdSe QDs and PEG-modified CdSe QDs on heart morphological development. Results displayed that CdSe QDs incubation caused obvious pericardial edema was $93 \%$ at 3 days post fertilization (3dpf) compared with the control group (Fig. 3A, B). While PEG-modified CdSe QDs significantly reduce the pericardial edema caused by CdSe QDs from 93-10\% (Fig. 3A, B). In addition, we found the CdSe QDs before and after modification did not have a serious effect on the heart rate (Supplementary Videos 1, 2, 3 and S6). Therefore, our results demonstrated that the CdSe QDs coated with PEG is an effective way to rescue the pericardial edema caused by CdSe QDs.

\subsection{Vascular toxicity before and after PEG camouflage for CdSe QDs}

Blood vessels are the transport channels of nanoparticles into the body. It has been reported that zebrafish embryos exposure to CdSe from 6 to $96 \mathrm{~h}$ exhibited vascular malformations. Also, CdSe QDs was highly toxic on the heart and when nanomaterials were injected intravenously, they quickly entered into blood vessel. Therefore, it is necessary to explore whether PEG-modified CdSe QDs can decreased the vascular toxicity of CdSe QDs. To visualize the vascular effects, the transgenic line $\mathrm{Tg}(K d r l$. mcherry) zebrafish with endothelial cells specific Kdrl promoter driving mcherry fluorescent was adopted. When vascular endothelial cells were affected, its expression decreased and showed vascular malformations. The whole images of zebrafish vascular endothelial cells showed that CdSe QDs incubation significantly reduce the expression intensity of vascular endothelial cells in both head and trunk (Fig. 4A-D). It is worth noting that CdSe QDs also lead to partial vessels missing (Fig. 4A, B; arrow indication). Yet, notable improvements in vascular marker expression and vascular integrity were observed for PEG-modified CdSe QDs in comparison with CdSe QDs (Fig. 4A-D). Vessel density analysis results indicated that the cardinal vein density significantly reduced by more than $50 \%$ in the CdSe QDs group compared the control group 
(Fig. 4E, F). It is worth noting that increased in the PEG-modified CdSe QDs increased about 32\% compared with CdSe QDs group (Fig. 4E, F). Besides, the intersegment vessel of zebrafish tail indicated abnormal bending or missing in the CdSe QDs group, which could be partly rescued in the PEG-modified CdSe QDs group (Figure S5). All these results suggested that PEG-modified CdSe QDs was an effective modification to reduce the influence of CdSe QDs on angiogenesis.

\subsection{Effect of CdSe QDs before and after PEG camouflage on inflammatory response}

A variety of materials will be treated as foreign bodies by the immune system after entering the body, the most typical is to activate the inflammatory response. We verified whether CdSe QDs caused inflammation in zebrafish embryos with the transgenic line Tg (coronin1a: EGFP) zebrafish embryos which the macrophages and neutrophils was marked by green fluorescent protein (GFP). Upon injury or infection to living tissues, inflammatory cells attracted by chemokines and cytokines are released from dead or dying cells and recruited to the injury site. As shown in Fig. 5, the expression intensity of macrophages and neutrophils in the CdSe QDs treatment group increased nearly twice (Fig. 5A-D). In addition, the number of macrophages and neutrophils increased sharply with CdSe QDs incubation (Fig. 5A-C). Compared with the CdSe QDs, the CdSe@PEG caused an obvious reduction in the macrophages and neutrophils intensity as well as number (Fig. 5A-D). These data demonstrated that PEG-modified CdSe QDs could alleviate the macrophages and neutrophil-mediated systemic inflammation in Tg (coronin 1a: EGFP) zebrafish triggered by CdSe QDs. This provides a new proof for reducing the immunotoxicity of nanomaterials.

\subsection{Neurotoxicity of CdSe QDs before and after PEG camouflage}

This is a common phenomenon that there is little mention of any neurotoxicity related safety verification in the massive papers in the field of materials. More recently, with the occurrence and development mechanism of neurological diseases gradually discovered, the potential nervous system damage caused by nanoparticles must be prudently monitored. To investigate whether CdSe QDs have the toxicity on neurons, we used the Tg (elav/3: Gal4vp16, UAS.mCherry) to detect the neurons expression, in which the pan-neuronal HuC/Elav3 promoter drives mCherry expression. When neurons damaged, the embryos exhibited reduced fluorescence expression and abnormal behavior. Results showed the neurons expression significantly reduced in the zebrafish head and spinal cord especially in midbrain and hindbrain in the CdSe QDs group compared with the control (Fig. 6A-D). While the midbrain and hindbrain neurons expression indicated the opposite trend in the CdSe@PEG group (Fig. 6A-D). In addition, the spinal cord neurons expression was obviously increased in CdSe@PEG group but not reach the control level (Fig. 6A-D). 
To further examine the influence of CdSe QDs and CdSe@PEG on the nervous system, we examined the behavior of zebrafish after different treatments. During the $30 \mathrm{~min}$ recording period, the trajectories of zebrafish in the CdSe QDs group were shorter and they tend to be more stationary while the trajectories were longer in the control and CdSe@PEG (Fig. 7A, C). In the heat map of the movement trajectory, it was more intuitive to find that the zebrafish in the CdSe QDs group prefer to be still, and the CdSe@PEG group reversed this phenomenon and the zebrafish became active (Fig. 7B). The statistics of moving speed (Fig. 7D),active time (Fig. 7E) and active frequency (Figure S7) and resting time (Figure S8) indicated that CdSe QDs significantly reduces the movement speed, active time and active frequency of zebrafish, which can be rescue by PEG-modified CdSe QDs. Taken together, PEG-modified ZnO QDs CdSe QDs could reduce CdSe QDs induced neurotoxicity and abnormal behavior.

\section{Conclusions}

In summary, we selected a series of nanomaterials, such as gold nanoparticles, upconversion nanomaterials and quantum dots, which are most commonly used in the diagnosis and treatment of diseases in vivo in recent years. The zebrafish embryos, which is a very sensitive animal model to toxins, is employed to scan their safety based on zebrafish mortality and hatching rate. Our results reveal that the toxicity of CdSe QDs is higher than that of gold nanoparticles and upconversion nanomaterials. In vivo organ toxicity test and analysis results showed CdSe QDs incubation caused pericardial edema, loss of vessels, decreased neuron expression and triggered intense inflammatory response. In order to reduce the side effects of nanomaterials, we choose some common modification methods. After CdSe QDs was encapsulated by silicon dioxide and the core-shell structure of CdSe@ZnS, the biocompatibility of CdSe QDs was significantly improved. Among these means of modification, the most biocompatible modification is polyethylene glycol (PEG) cladding method. In order to explore the improvement of CdSe QDs toxicity after PEG camouflage, a series of transgenic zebrafish embryos were employed to evaluate the toxicity of CdSe QDs from these aspects of heart, blood vessel, immune system and nervous system. The results manifested CdSe@PEG could significantly increase the survival and hatching rate of zebrafish embryos as well as reduce the malformation rate compared with no camouflage group. PEGcamouflaged CdSe QDs was a more effective method to minimize the toxicity including the negative effects on the heart, nerves, blood vessels and inflammation. These nanomaterials in our work are randomly selected, and with this scanning method for embryo safety, any material of interest can be replaced, such as 2D metal carbides and nitrides (MXenes) and metal-organic frameworks (MOFs). We will continue to study the impact of nanomaterials on other tissue and organs, and further try to exchange for different polymer modifications for screening and will study the mechanism of the influence of nanomaterials on each system. Our work expects to inspire the more comprehensive evaluation strategies of biocompatibility for biomedical nanoparticles, especially in nerves, heart and blood vessels of embryos that are particularly sensitive to harmful substances.

\section{Materials And Methods}


Zebrafish lines and maintenance. Unless otherwise stated, the wild-type (Tu strain) embryos were used for experiments. The transgenic lines of $\mathrm{Tg}$ (kdrl: mcherry), $\mathrm{Tg}$ (coronin 1a: EGFP) and $\mathrm{Tg}$ (elavl3:Gal4vp16,UAS.mcherry) line crossed with wild type respectively, and the transgenic embryos are selected for analysis of the vascular toxicity, inflammatory toxicity and the neurotoxicity. $\mathrm{Tg}$ (kdrl:mCherry) ${ }^{29}$ were obtained from Dr. Bo Zhang (School of Life Science, Peking University, Beijing, China). The Tg (coronin 1a: EGFP) line was provided by Dr Zilong Wen (Hong Kong University of Science and Technology, Hong Kong, China $)^{30}$ and Tg(elav/3: Gal4vp16,UAS.mcherry) line were kindly provided by Dr. Jiulin Du (Institute of Neuroscience, University of Chinese Academy of Sciences, Shanghai, China). All protocols for animal procedures were approved by the Animal Ethics Committee of Tianjin University.

Different nanomaterial treatment. Select normal development embryos at $4 \mathrm{hpf}$ under a stereomicroscope and place them in a 12-well plate (20 embryos in a well). The embryos were divided into 6 groups and one group with 3 parallel wells. The embryos were incubated in E3 culture medium with different concentrations of Au NPs, UCNPs, C QDs, ZnO QDs and CdSe QDs from $4 \mathrm{hpf}$ to $72 \mathrm{hpf}$. In the nanomaterial modification screening experiment, the embryos were incubated in E3 culture medium with $0.1 \mathrm{mg} / \mathrm{ml} \mathrm{SiO}_{2}$-modified CdSe QDs, ZnS-modified CdSe QDs and PEG-modified of CdSe QDs from $4 \mathrm{hpf}$ to $72 \mathrm{hpf} .$. The mortality and deformity rates were recorded every day. Embryos hatching rate, body bending rate, pericardial edema rate were analyzed after nanomaterial treatment at $3 \mathrm{dpf}$.

In vivo study the effect of CdSe QDs and PEG-modified of CdSe QDs on heart. The wild type embryos were incubated in E3 culture medium and E3 with CdSe QDs and PEG-modified of CdSe QDs from $4 \mathrm{hpf}$ to $72 \mathrm{hpf}$. After the removal of nanomaterials, the pericardial edema and heart rate were imaged by Nikon Ti2-U.

In vivo study the effect of CdSe QDs and PEG-modified of CdSe QDs on vascular. The heterozygote $\mathrm{Tg}$ ( $k d r l$ : mcherry) embryos were incubated in E3 culture medium and E3 with CdSe QDs and PEG-modified of CdSe QDs from $4 \mathrm{hpf}$ to $72 \mathrm{hpf}$. After the removal of nanomaterials, the expression of vascular endothelial cells in control, CdSe QDs and CdSe@PEG group were imaged respectively by Leica THUNDER Imager Model Organism. The fluorescence intensity was analyzed by LAS X software. The vessel branch was analyzed with Image J Angiogenesis Analyze.

In vivo study the effect of CdSe QDs and PEG-modified of CdSe QDs on inflammatory response. The heterozygote $\mathrm{Tg}$ (coronin 1a: EGFP) embryos were incubated in E3 culture medium and E3 with CdSe QDs and PEG-modified of CdSe QDs from $4 \mathrm{hpf}$ to $72 \mathrm{hpf}$. After the removal of nanomaterials, the expression of macrophages and neutrophils in control, CdSe QDs and CdSe@PEG group were imaged respectively by Leica THUNDER Imager Model Organism. The fluorescence intensity was analyzed by LAS X software.

In vivo study the effect of CdSe QDs and PEG-modified of CdSe QDs on nerve.The heterozygote $\mathrm{Tg}$ (elav/3: mCherry) embryos were incubated in E3 culture medium and E3 with CdSe QDs and PEG-modified of CdSe QDs from $4 \mathrm{hpf}$ to $72 \mathrm{hpf}$. After the removal of nanomaterials, the expression of neurons in 
control, CdSe QDs and CdSe@PEG group were imaged respectively by Leica THUNDER Imager Model Organism. The fluorescence intensity was analyzed by LAS X software.

Zebrafish larvae behavioral analysis.The wild type embryos were incubated in E3 culture medium and E3 with CdSe QDs and PEG-modified of CdSe QDs from $4 \mathrm{hpf}$ to $72 \mathrm{hpf}$. After the removal of nanomaterials, the embryos were place in the 24-well plate for behavioral analysis with DanioVision.

Statistical Analysis. Data were expressed as mean \pm standard deviation (SD) of experiments. Data analysis was performed using Origin 8.0. The significance between groups was analyzed using an unpaired two-tailed t test (comparing two groups) and one-way analysis of variance (ANOVA) (comparing multiple groups) by Statistics Analysis System ( ${ }^{*} P<0.05$, ${ }^{\star *} \mathrm{P}<0.01$ and $\left.{ }^{* \star *} \mathrm{P}<0.001\right)$. $\mathrm{P}<0.05$ was considered significant.

\section{Declarations}

\section{Ethical Approval and Consent to participate}

All animal experiments are approved by Tianjin University and consent to participate.

\section{Consent for publication}

Not applicable.

\section{Availability of supporting data}

All supporting data is available.

\section{Competing interests}

The authors declare that they have no competing interests.

\section{Authors' contributions}

${ }^{1}$ The same contribution.

\section{Acknowledgement}

The authors thank Drs. Li Yuhao and Cui Jianlin for assistance with zebrafish space and experimental equipment, Drs. Xiong Jingwei and Zhang bo for assistance with transgenic lines. We thank the instrument analytical center of School of Pharmaceutical Science and Technology at Tianjin University for providing the Stereo Fluorescence Microscope assistance with Mr. Zhi Li.

\section{Funding}


This work supported by the National Natural Science Foundation of China (32000999), Key Project of Tianjin Natural Science Foundation (19JCZDJC34100), and the National Key Research and Development Program of China (2017YFB1300302).

\section{Authors' information}

All authors form Academy of Medical Engineering and Translational Medicine, Tianjin University, 92 Weijin Road, Nankai District, Tianjin 300072, P.R. China.

\section{References}

[1] M. Gatoo, S. Naseem, M. Arfat, A. Dar, K. Qasim, S. Zubair, Physicochemical Properties of Nanomaterials: Implication in Associated Toxic Manifestations, Biomed Res. Int. 2014 (2014) 498420.

[2] M. Parani, G. Lokhande, A. Singh, A.K. Gaharwar, Engineered Nanomaterials for Infection Control and Healing Acute and Chronic Wounds, ACS Appl. Mater. Interfaces. 8 (2016) 10049-10069.

[3] M. Mohajeri, B. Behnam, G.E. Barreto, A. Sahebkar, Carbon Nanomaterials and Amyloid-Beta Interactions: Potentials for the Detection and Treatment of Alzheimer's Disease? Pharmacol. Res. 143 (2019) 186-203.

[4] T. Liu, B. Xiao, F. Xiang, J. Tan, Z. Chen, X. Zhang, C. Wu, Z. Mao, G. Luo, X. Chen, J. Deng, Ultrasmall Copper-Based Nanoparticles for Reactive Oxygen Species Scavenging and Alleviation of Inflammation Related Diseases, Nat. Commun. 11 (2020) 2788.

[5] J. Luan, A. Seth, R. Gupta, Z. Wang, P. Rathi, S. Cao, D.H. Gholami, R. Tang, B. Xu, S. Achilefu, J.J. Morrissey, S. Singamaneni, Ultrabright Fluorescent Nanoscale Labels for the Femtomolar Detection of Analytes with Standard Bioassays, Nat. Biomed. Eng. 4 (2020) 518-530.

[6] M. Ovais; S. Mukherjee, A. Pramanik, D. Das, A. Mukherjee, A. Raza, C. Chen, Designing Stimuliresponsive Upconversion Nanoparticles that Exploit the Tumor Microenvironment, Adv. Mater. 32 (2020) 2000055.

[7] S. Pleskova, E. Mikheeva, E. Gornostaeva, Using of Quantum Dots in Biology and Medicine, Adv. Exp. Med. Biol. 1048 (2018) 323-334.

[8] C.T. Matea, T. Mocan, F. Tabaran, T. Pop, O. Mosteanu, C. Puia, C. lancu, L. Mocan, Quantum Dots in Imaging, Drug Delivery and Sensor Applications, Int. J. Nanomedicine. 28 (2017) 5421 - 5431.

[9] N. Lewinski, V. Colvin D, R. rezek, Cytotoxicity of Nanoparticles, Small 4(2008) 26 - 49.

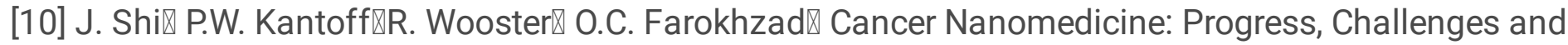
Opportunities. Nat. Rev. Cancer. 17 (2017) 20 - 37. 
[11] D. Sun, G. Qi, K. Ma, X. Qu, W. Xu, S. Xu, Y. Jin, Tumor Microenvironment-Activated Degradable Multifunctional Nanoreactor for Synergistic Cancer Therapy and Glucose SERS Feedback. iScience 23 (2020) 101274.

[12] T. Komatsu, E. Kyo, H. Ishii, K. Tsuchikama, A. Yamaguchi, T. Ueno, K. Hanaoka, Y. Urano, Antibody Clicking as a Strategy to Modify Antibody Functionalities on the Surface of Targeted Cells, J. Am. Chem. Soc. 142 (2020) 15644 - 15648.

[13] C. Pan, J. Wu, S. Qing, X. Zhang, L. Zhang, H. Yue, M. Zeng, B. Wang, Z. Yuan, Y. Qiu, H. Ye, D. Wang, X. Liu, P. Sun, B. Liu, E. Feng, X. Gao, Zhu, W. Wei, G. Ma, H. Wang, Biosynthesis of Self-Assembled Proteinaceous Nanoparticles for Vaccination, Adv. Mater. 2 (2020) 2002940.

[14] Z. Cao, X. Wang, Y. Pang, S. Cheng, J. Liu, Biointerfacial Self-Assembly Generates Lipid Membrane Coated Bacteria for Enhanced Oral Delivery and Treatment, Nat. Commun. 10 (2019) 5783.

[15] P. Feng, Z. Cao, X. Wang; J. Li, J. Liu, On-Demand Bacterial Reactivation by Restraining within a Triggerable Nanocoating, Adv. Mater. 32 (2020) 2002406.

[16] F. Campbell, F.L. Bos, S. Sieber, G. Arias-Alpizar, B.E. Koch, J. Huwyler, A. Kros, J. Bussmann, Directing Nanoparticle Biodistribution through Evasion and Exploitation of Stab2-Dependent Nanoparticle Uptake, ACS Nano 12 (2018) 2138 - 2150.

[17] I.J. Marques, E. Lupi, N. Mercader, Model Systems for Regeneration: Zebrafish, Development 146 (2019) 167692.

[18] K.D. Poss, L.G. Wilson, M.T. Keating, Heart Regeneration in Zebrafish, Science 298 (2002) 2188 2190.

[19] C. Xiao, L. Gao, Y. Hou, C. Xu, N. Chang, F. Wang, K. Hu, A. He, Y. Luo, J. Wang, J. Peng, F. Tang, X. Zhu, J.W. Xiong, Chromatin-Remodelling Factor Brg1 regulates Myocardial Proliferation and Regeneration in Zebrafish, Nat. Commun. 7 (2016) 13787.

[20] W. Li, Y. Zhang, B. Han, L. Li, M, Li, X. Lu, C. Chen, M. Lu, Y. Zhang, X. Jia, Z. Zhu, X. Tong, B. Zhang, One-Step Efficient Generation of Dual-Function Conditional Knockout and Geno-Tagging Alleles in Zebrafish, Elife 8 (2019) 48081.

[21] N. Chang, C. Sun, L. Gao, D. Zhu, X. Xu, X. Zhu, J.W. Xiong, J.J. Xi, Genome Editing with RNA-Guided Cas9 Nuclease in Zebrafish Embryos, Cell Res. 23 (2013) 465 - 472.

[22] Z. Ma, P. Zhu, M. Pang, L. Guo, N. Chang, J. Zheng, X. Zhu, C. Gao, H. Huang, Z. Cui, J.W. Xiong, J. Peng, J. Chen, A Novel Inducible Mutagenesis Screen Enables to Isolate and Clone both Embryonic and Adult Zebrafish Mutants, Sci. Rep. 7 (2017) 10381. 
[23] K.R. Astell囚D. Sieger Zebrafish In Vivo Models of Cancer and Metastasis $₫$ Cold Spring Harb. Perspect. Med. 10 (2020) 037077.

[24] Y. Ding, J. Ma, A.D. Langenbacher, K.I. Baek, J. Lee, C.C. Chang, J.J. Hsu, R.P. Kulkarni, J. Belperio, W. Shi, S. Ranjbarvaziri, R. Ardehali, Y. Tintut, L.L. Demer, J.N. Chen, P. Fei, R.R.S. Packard, T.K. Hsiai, Multiscale Light-Sheet for Rapid Imaging of Cardiopulmonary System, JCI Insight 3 (2018) 121396.

[25] K. Kawakami, D.A. Largaespada, Z. Ivics, Transposons as Tools for Functional Genomics in Vertebrate Models, Trends Genet. 33 (2017) 784 - 801.

[26] S. Bae, M. Park, C. Kang, S. Dilmen, T.H. Kang, D.G. Kang, Q. Ke, S.U. Lee, D. Lee, P.M. Kang, Hydrogen Peroxide-Responsive Nanoparticle Reduces Myocardial Ischemia/Reperfusion Injury, J Am Heart Assoc. 5 (2016) 003697.

[27] B.P. Sampurna, F. Santoso, J.H. Lee, W.H. Yu, C.C. Wu, G. Audira, S. Juniardi, J.R. Chen, Y.T. Lin, C.D. Hsiao, Cardiac Rhythm and Molecular Docking Studies of Ion Channel Ligands with Cardiotoxicity in Zebrafish, Cells 8 (2019) 566.

[28] Y. Han, B. Chen, J. Zhang, C. Hu, Cardiac Safety Evaluation in Zebrafish and in Silico ADME Prediction of Cephalosporins with an Aminothiazoyl Ring at the C-7 Position, Toxicol. Appl. Pharmacol. 347 (2018) 33 - 44.

[29] Z. Xia, X. Tong, F. Liang, Y. Zhang, C. Kuok, Y. Zhang; X. Liu, Z. Zhu, S. Lin, B. Zhang, Eif3ba Regulates Cranial Neural Crest Development by Modulating p53 in Zebrafish, Dev. Biol. 381 (2013) 83 - 96.

[30] Y.J. Li, B. Hu, Establishment of Multi-site Infection Model in Zebrafish Larvae for Studying Staphylococcus Aureus Infectious Disease, J. Genet. Genomics 39 (2012) 521 - 534.

[31] B.A. Maher, I.A. Ahmed,V. Karloukovski, D.A. MacLaren, P.G. Foulds, D. Allsop, D.M. Mann, R. TorresJardón, L. Calderon-Garciduenas, Magnetite Pollution Nanoparticles in the Human Brain, Proc. Natl. Acad. Sci. USA. 113 (2016) 10797 - 10801.

[32] W.K. Boyes, T.C. Van, Neurotoxicology of Nanomaterials, Chem. Res. Toxicol. 33 (2020) 1121 - 1144.

\section{Figures}



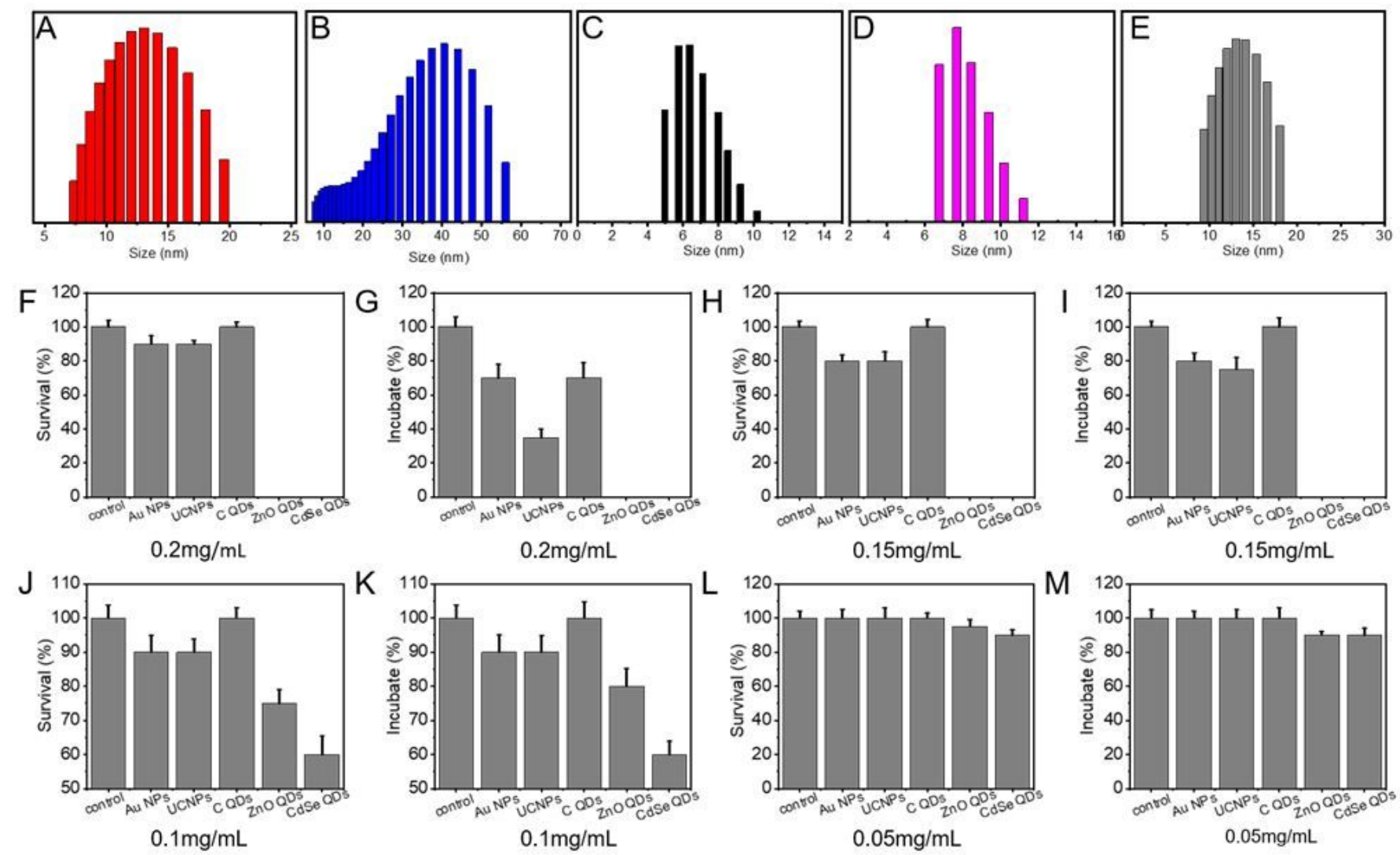

$0.15 \mathrm{mg} / \mathrm{mL}$

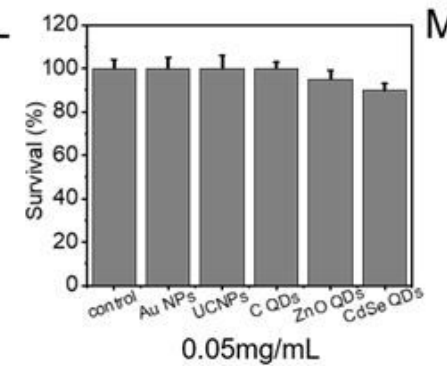

$0.15 \mathrm{mg} / \mathrm{mL}$

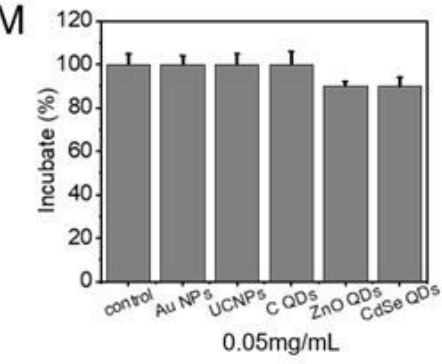

Figure 1

Toxicity detection of Au NPs, UCNPs, C QDs, ZnO QDs, CdSe QDs. (A, B, C, D, E) DLS of Au NPs, UCNPs, C QDs, ZnO QDs, CdSe QDs. (F, G) Survival rate and hatch rate of zebrafish embryos with $0.2 \mathrm{mg} / \mathrm{mL}$ Au NPs, UCNPs, C QDs, ZnO QDs, CdSe QDs. $(\mathrm{H}, \mathrm{l})$ The survival rate and hatching rate of zebrafish embryos soaked at a concentration of $0.15 \mathrm{mg} / \mathrm{mL}$ Au NPs, UCNPs, C QDs, ZnO QDs, CdSe QDs. (J, K) The survival rate and hatching rate of zebrafish embryos soaked at a concentration of $0.1 \mathrm{mg} / \mathrm{mL}$ Au NPs, UCNPs, C QDs, ZnO QDs, CdSe QDs. (L, M) The survival rate and hatching rate of zebrafish embryos with $0.05 \mathrm{mg} / \mathrm{mL}$ Au NPs, UCNPs, C QDs, ZnO QDs, CdSe QDs, n=20. 

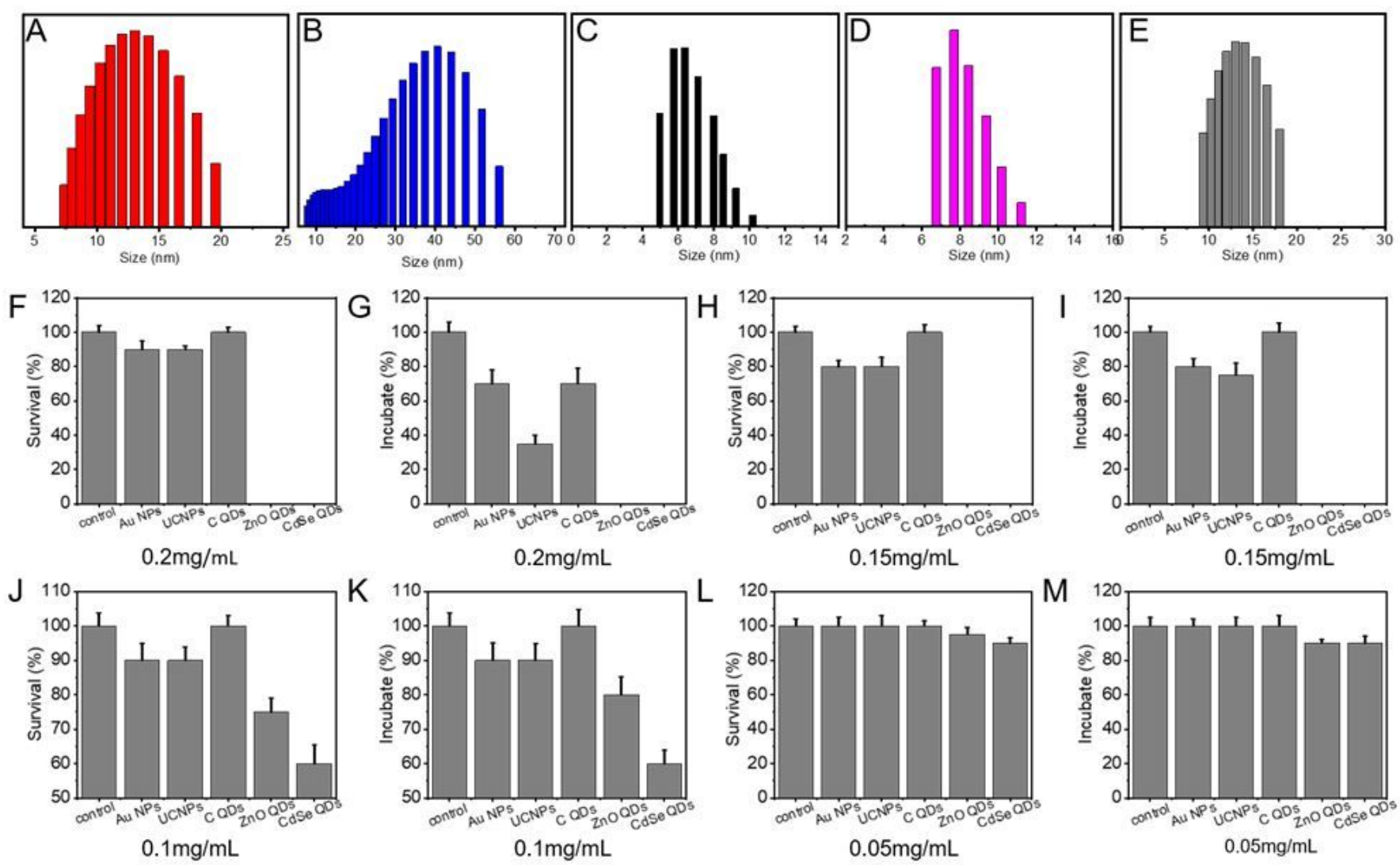

$0.15 \mathrm{mg} / \mathrm{mL}$

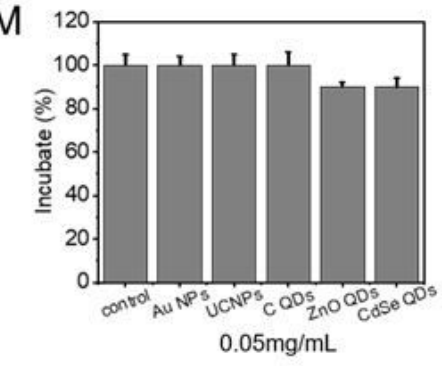

\section{Figure 1}

Toxicity detection of Au NPs, UCNPs, C QDs, ZnO QDs, CdSe QDs. (A, B, C, D, E) DLS of Au NPs, UCNPs, C QDs, ZnO QDs, CdSe QDs. $(F, G)$ Survival rate and hatch rate of zebrafish embryos with $0.2 \mathrm{mg} / \mathrm{mL}$ Au NPs, UCNPs, C QDs, ZnO QDs, CdSe QDs. $(\mathrm{H}, \mathrm{I})$ The survival rate and hatching rate of zebrafish embryos soaked at a concentration of $0.15 \mathrm{mg} / \mathrm{mL}$ Au NPs, UCNPs, C QDs, ZnO QDs, CdSe QDs. (J, K) The survival rate and hatching rate of zebrafish embryos soaked at a concentration of $0.1 \mathrm{mg} / \mathrm{mL}$ Au NPs, UCNPs, C QDs, ZnO QDs, CdSe QDs. (L, M) The survival rate and hatching rate of zebrafish embryos with 0.05mg/mL Au NPs, UCNPs, C QDs, ZnO QDs, CdSe QDs, n=20. 

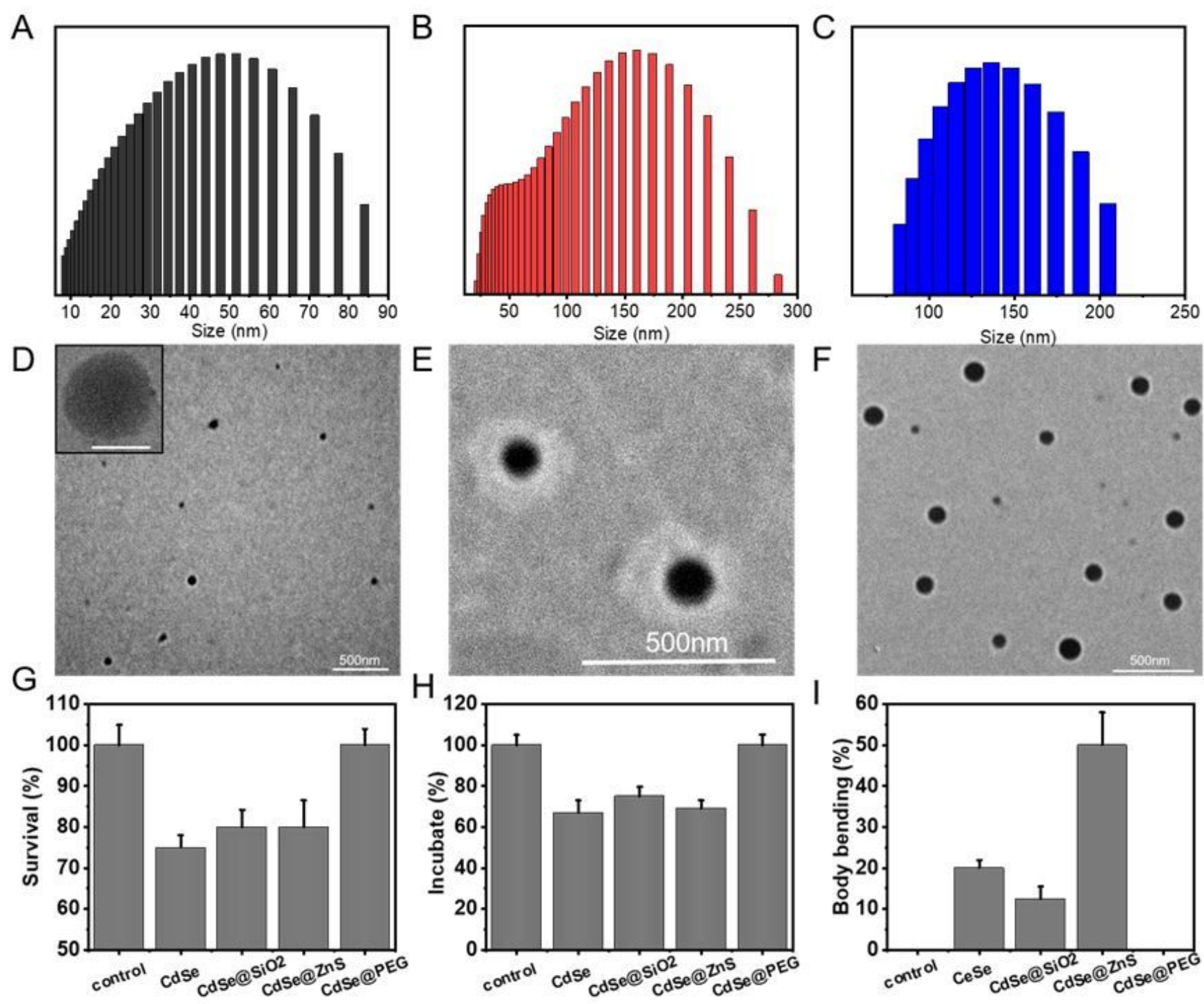

Figure 2

Toxicity of different modification methods of CdSe QDs to zebrafish. (A, B, C) DLS of CdSe@SiO2, CdSe@ZnS, CdSe@PEG. (D, E, F) TEM images of CdSe@SiO2, CdSe@ZnS, CdSe@PEG. The zebrafish embryos survival rate. $(\mathrm{G})$, hatching rate. $(\mathrm{H})$ Body bending rate in CdSe@SiO2, CdSe@ZnS and CdSe@PEG groups. 

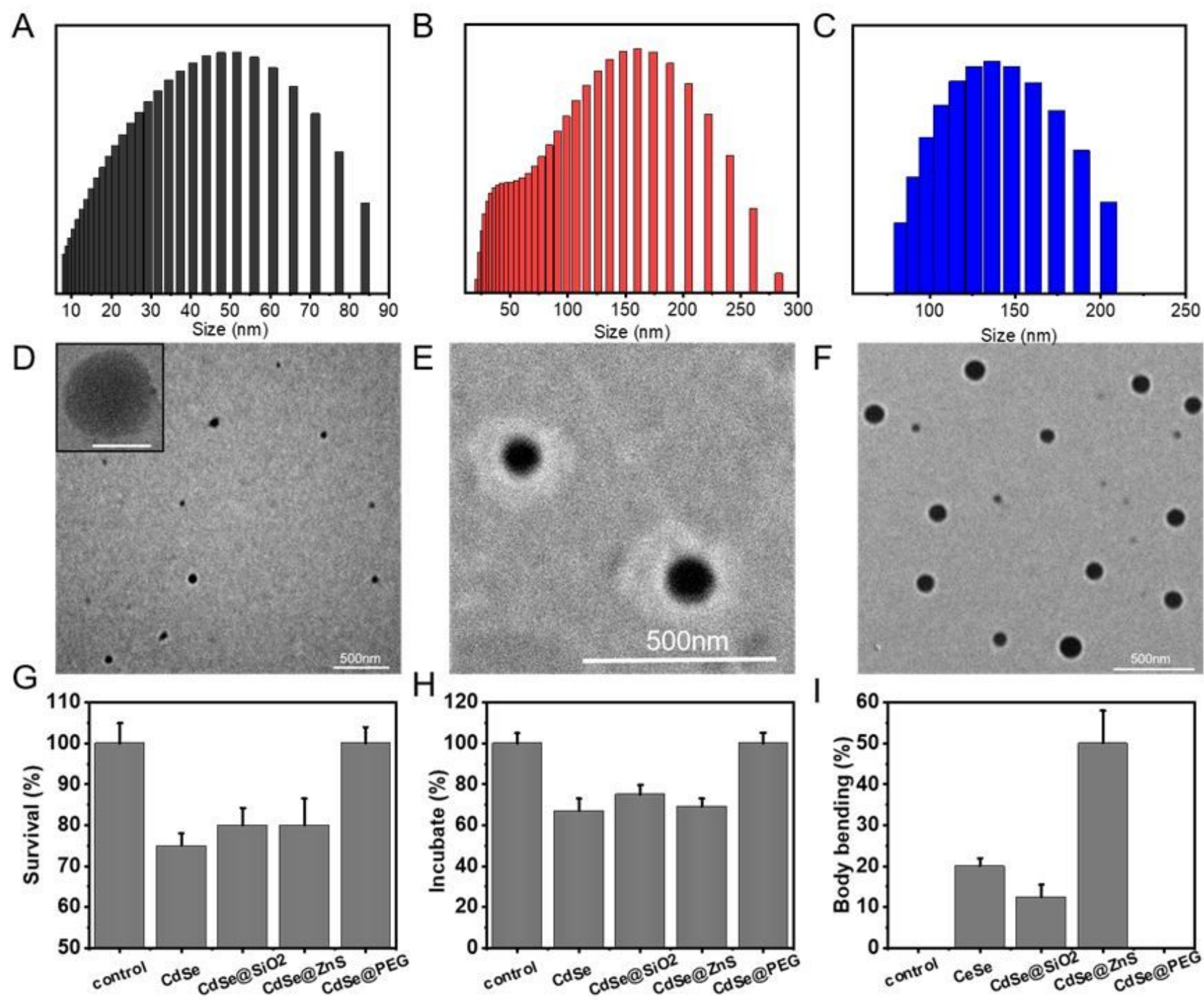

Figure 2

Toxicity of different modification methods of CdSe QDs to zebrafish. (A, B, C) DLS of CdSe@SiO2, CdSe@ZnS, CdSe@PEG. (D, E, F) TEM images of CdSe@SiO2, CdSe@ZnS, CdSe@PEG. The zebrafish embryos survival rate. $(\mathrm{G})$, hatching rate. $(\mathrm{H})$ Body bending rate in CdSe@SiO2, CdSe@ZnS and CdSe@PEG groups. 

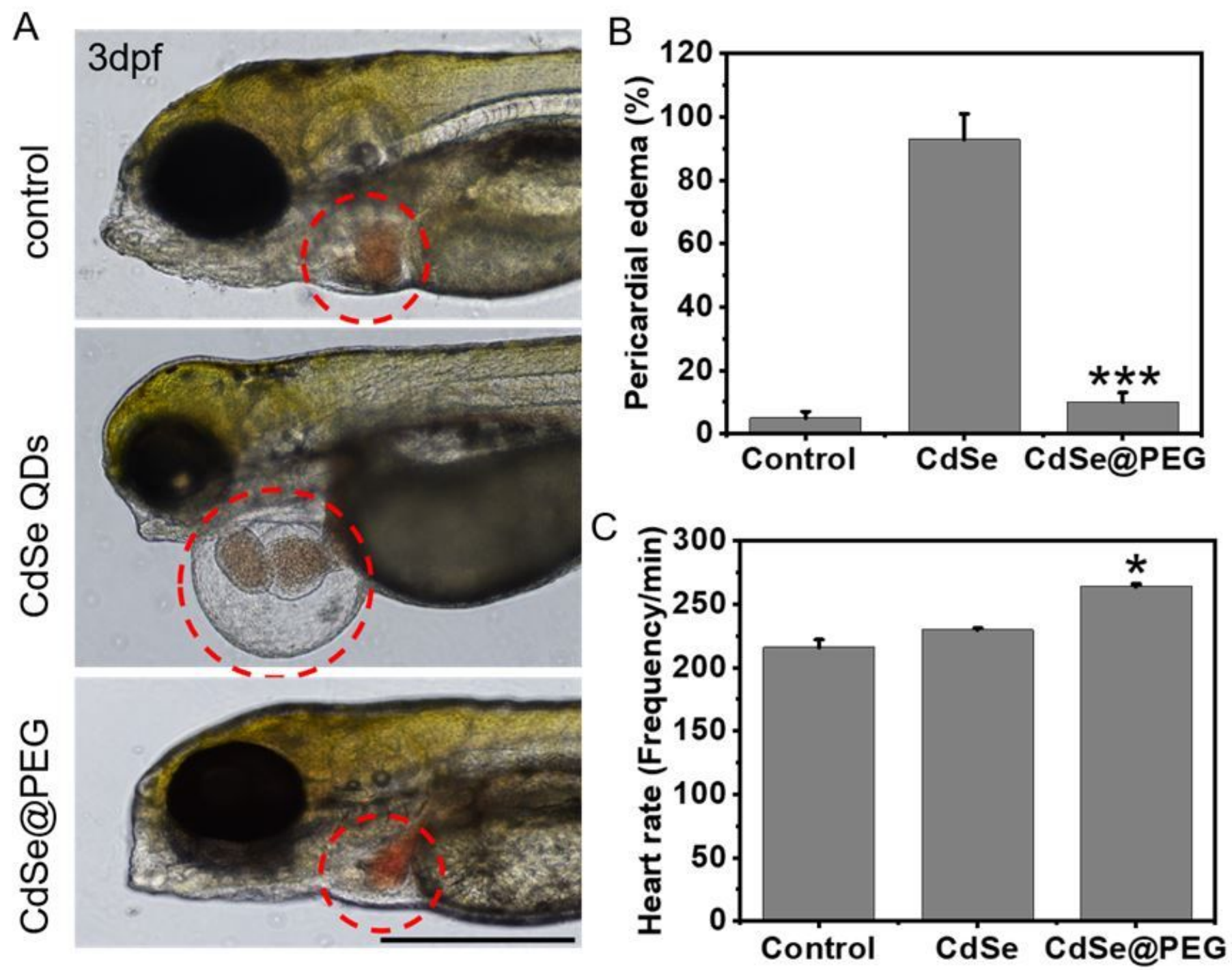

Figure 3

PEG-modified significantly reduced the heart toxicity of CdSe QDs in zebrafish. (A) The heart image of zebrafish in control, CdSe QDs and CdSe@PEG groups. (B) The pericardial edema rate of zebrafish in control, CdSe QDs and CdSe@PEG groups. (C) The heart rate of zebrafish in control, CdSe QDs and CdSe@PEG groups. n=20. Scale bars: $500 \mu m$. 

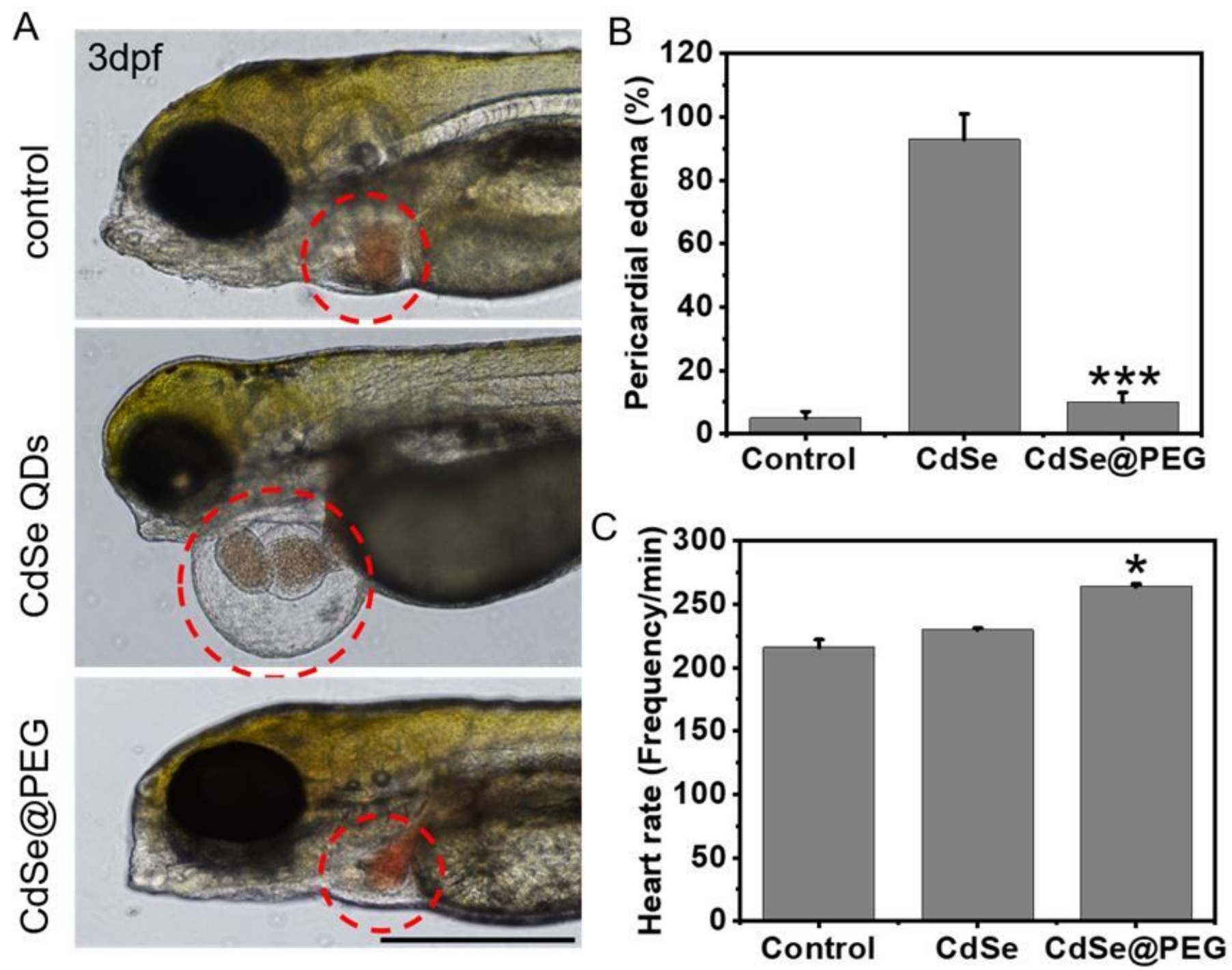

Figure 3

PEG-modified significantly reduced the heart toxicity of CdSe QDs in zebrafish. (A) The heart image of zebrafish in control, CdSe QDs and CdSe@PEG groups. (B) The pericardial edema rate of zebrafish in control, CdSe QDs and CdSe@PEG groups. (C) The heart rate of zebrafish in control, CdSe QDs and CdSe@PEG groups. n=20. Scale bars: $500 \mu m$. 


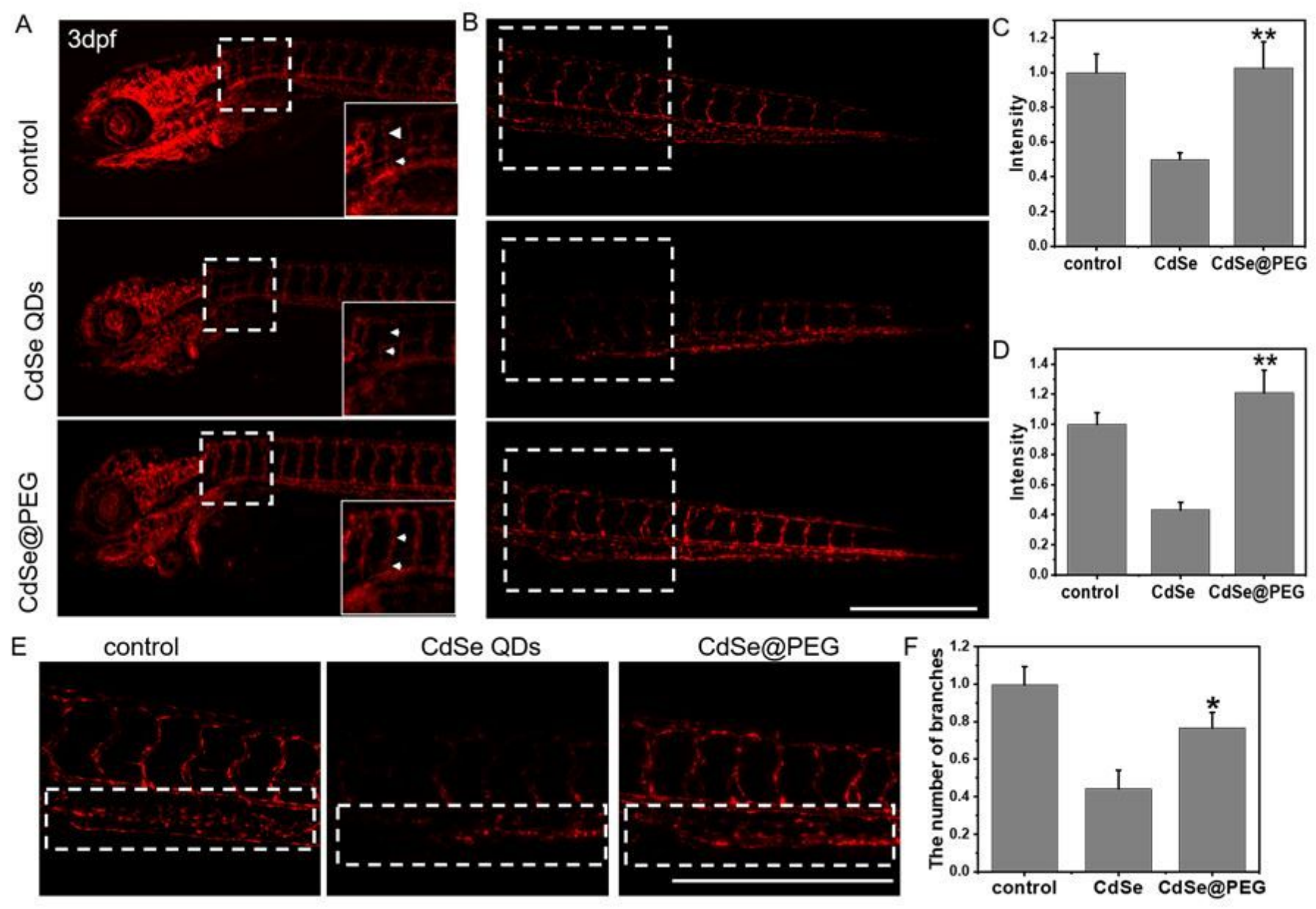

Figure 4

PEG-modified significantly decreased the vessels toxicity of CdSe QDs in zebrafish. (A, C) The zebrafish head vascular endothelial cells expression and fluorescence quantification in control, CdSe QDs and CdSe@PEG groups. (B, D) The zebrafish trunk vascular endothelial cells expression and fluorescence quantification in control, CdSe QDs and CdSe@PEG groups. (E) Enlarged view of dotted frame in B. (F) The cardinal vein density analysis in control, CdSe QDs and CdSe@PEG groups. n=20. Scale bars: $500 \mu \mathrm{m}$. 


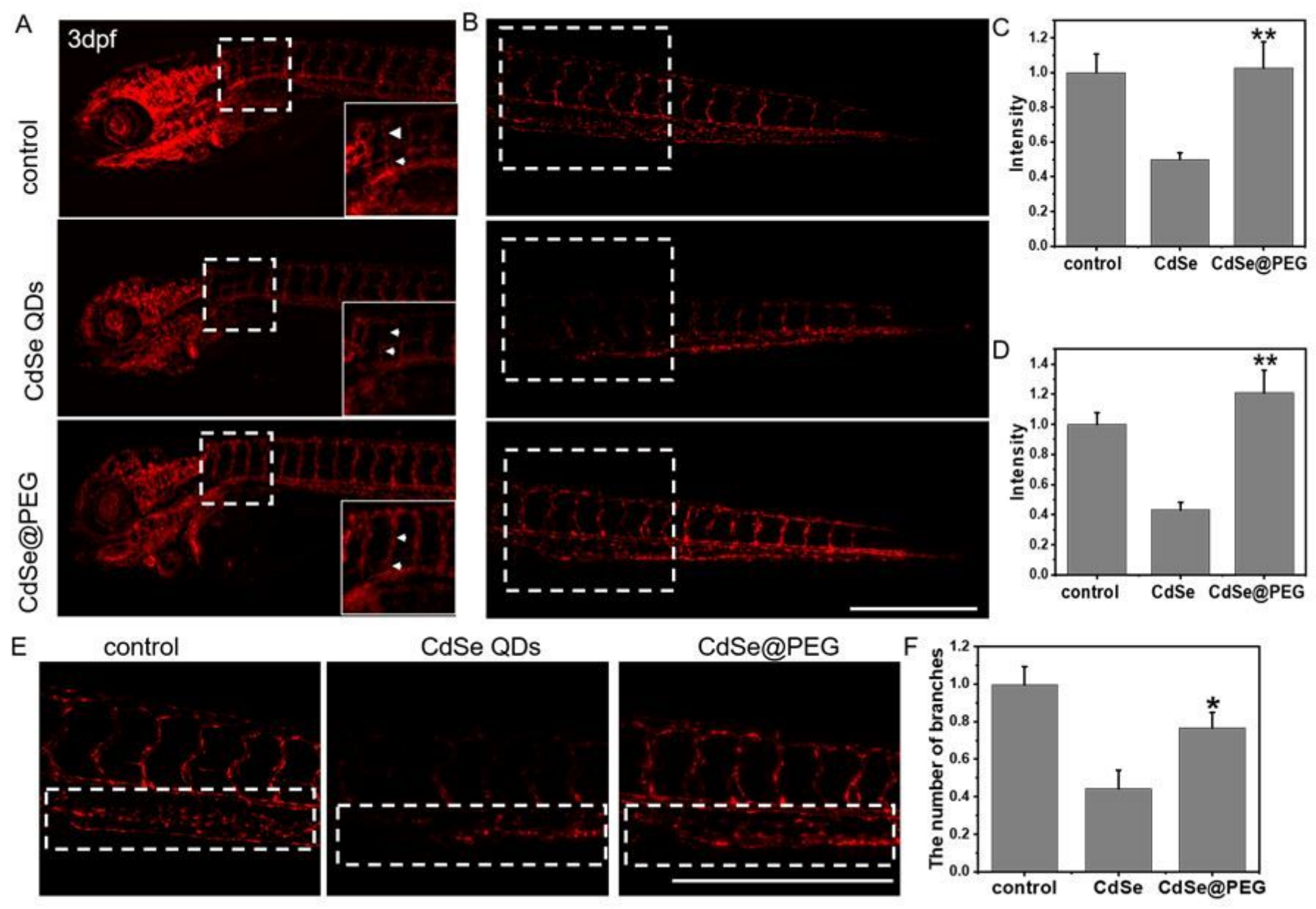

Figure 4

PEG-modified significantly decreased the vessels toxicity of CdSe QDs in zebrafish. (A, C) The zebrafish head vascular endothelial cells expression and fluorescence quantification in control, CdSe QDs and CdSe@PEG groups. (B, D) The zebrafish trunk vascular endothelial cells expression and fluorescence quantification in control, CdSe QDs and CdSe@PEG groups. (E) Enlarged view of dotted frame in B. (F) The cardinal vein density analysis in control, CdSe QDs and CdSe@PEG groups. n=20. Scale bars: $500 \mu \mathrm{m}$. 

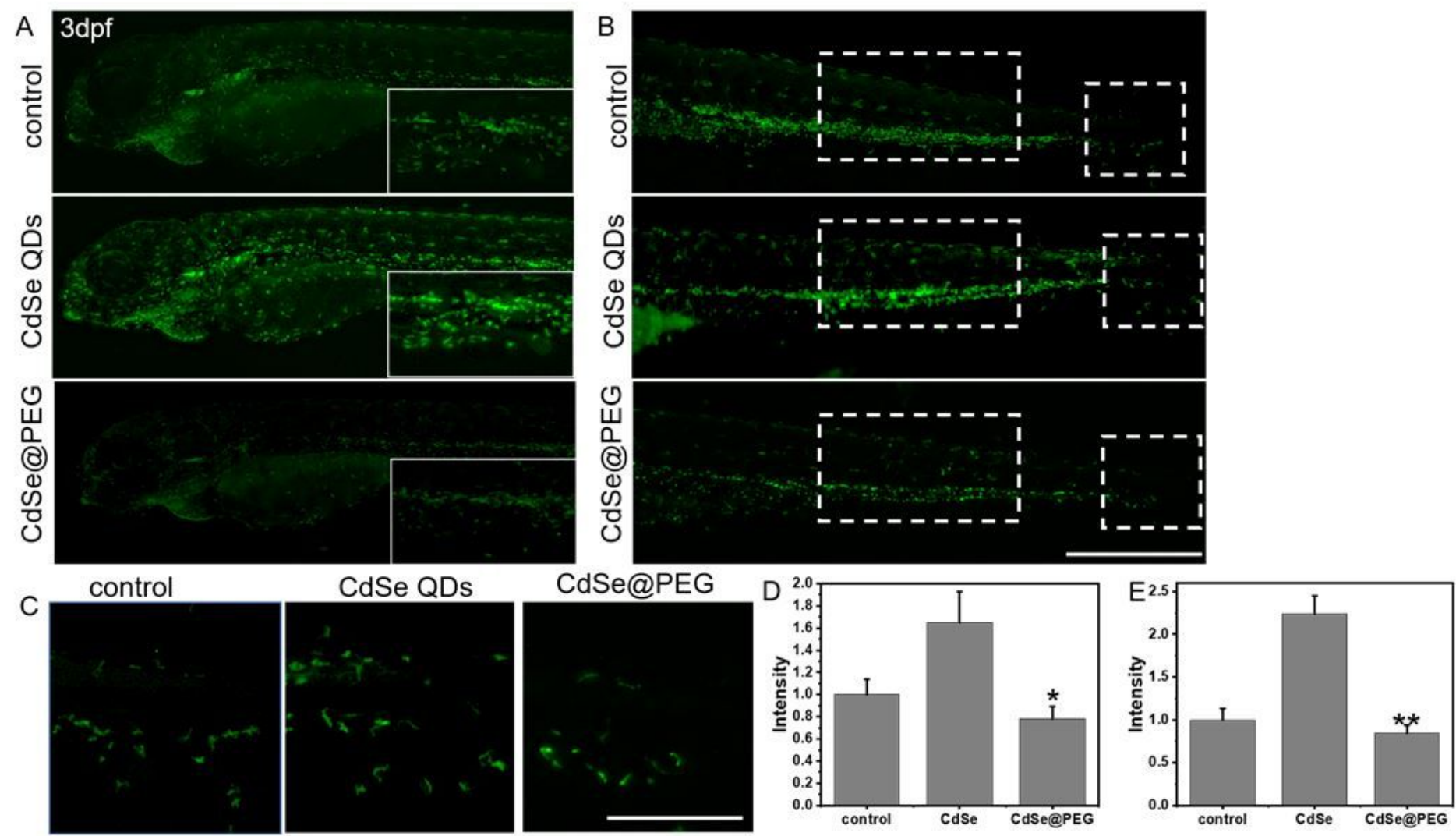

Figure 5

PEG-modified significantly decreased the inflammation response of CdSe QDs in zebrafish. (A, B) The effect of CdSe QDs, CdSe@PEG on inflammatory cells in the head of zebrafish and trunk. (C) Enlarged view of dotted frame of tail in $B$, white dotted circle marked macrophages and neutrophils. (D, E) Analysis of the intensity of inflammatory cells expression of zebrafish head and trunk in control, CdSe QDs and CdSe@PEG groups. n=20. Scale bars: $500 \mu \mathrm{m}$. 

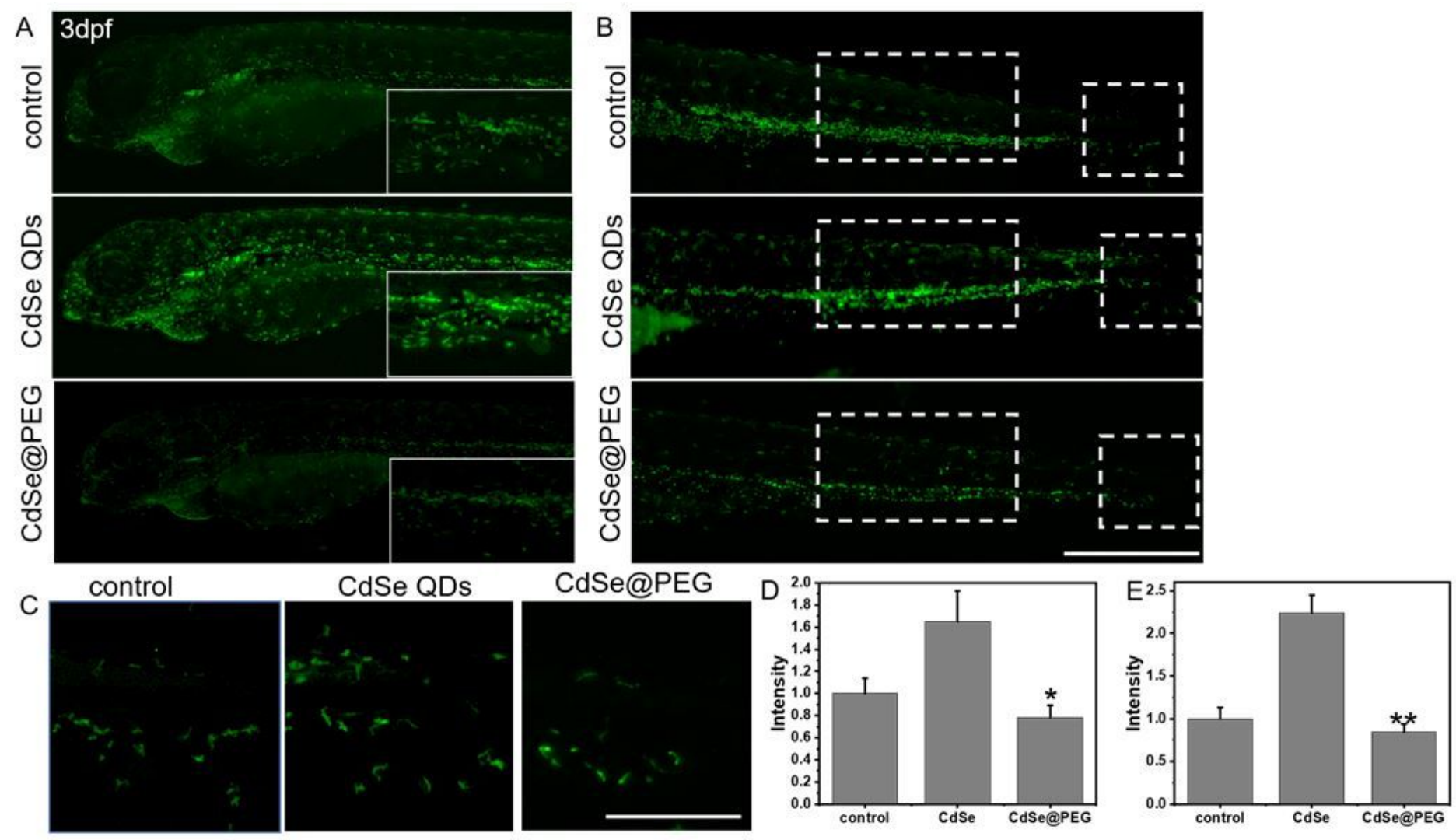

Figure 5

PEG-modified significantly decreased the inflammation response of CdSe QDs in zebrafish. (A, B) The effect of CdSe QDs, CdSe@PEG on inflammatory cells in the head of zebrafish and trunk. (C) Enlarged view of dotted frame of tail in $B$, white dotted circle marked macrophages and neutrophils. (D, E) Analysis of the intensity of inflammatory cells expression of zebrafish head and trunk in control, CdSe QDs and CdSe@PEG groups. n=20. Scale bars: $500 \mu \mathrm{m}$. 


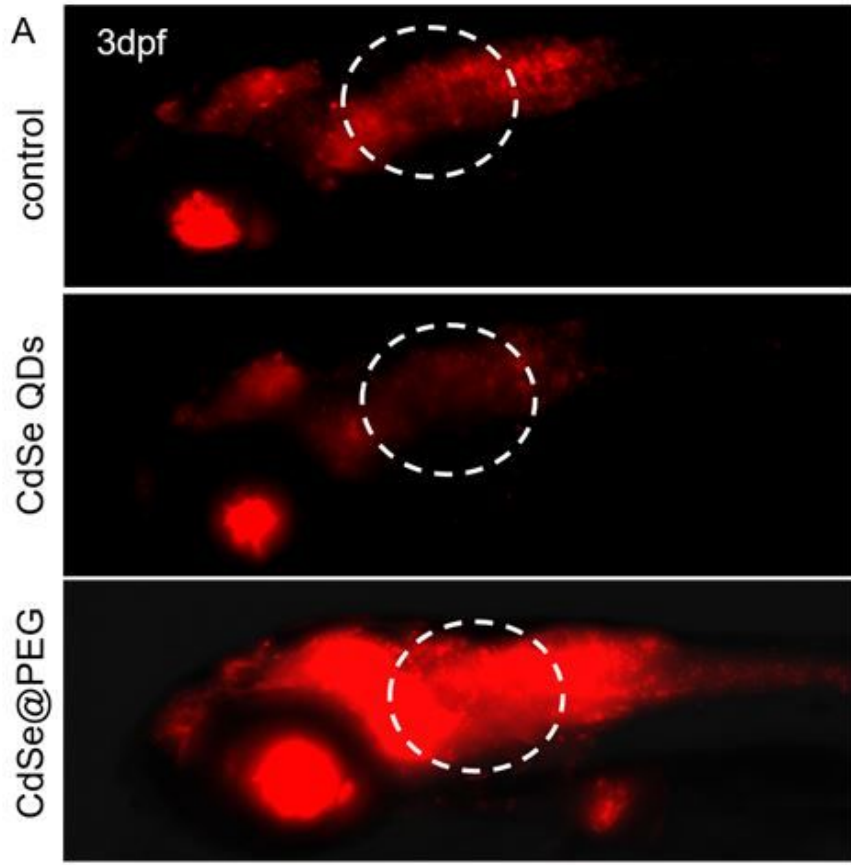

C

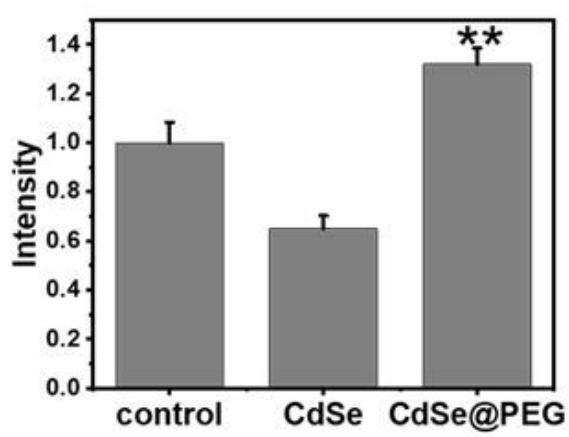

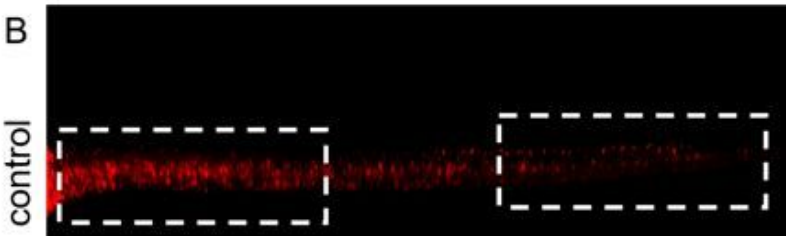
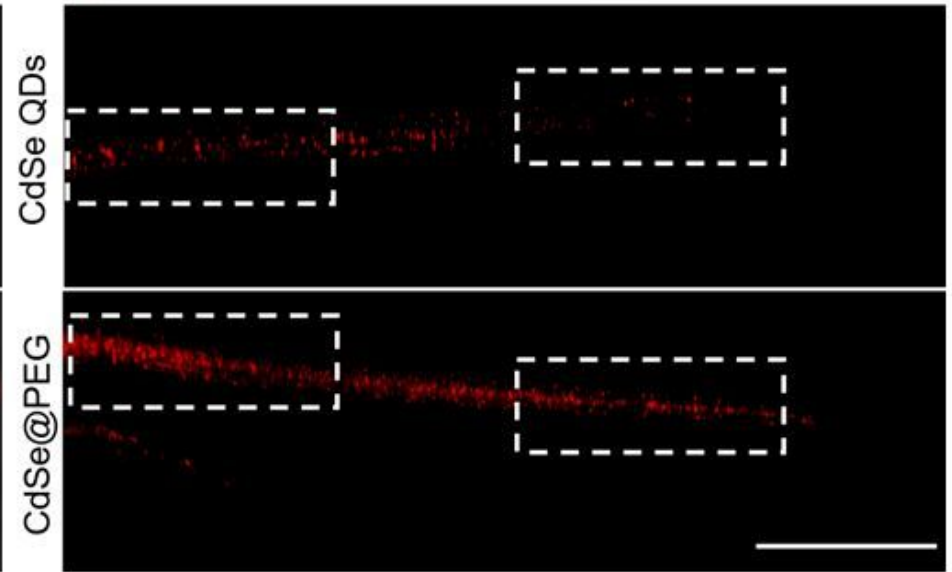

D

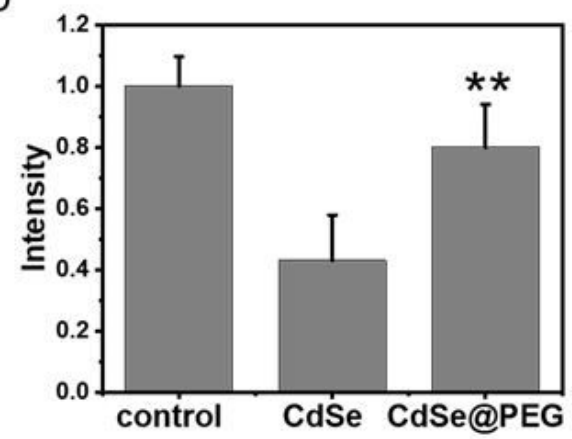

Figure 6

PEG-modified significantly decreased the nerve toxicity of CdSe QDs in zebrafish. (A, B) The effect of CdSe QDs, CdSe@PEG on neurons in zebrafish head and trunk. (C, D) Analysis of the intensity of neurons expression of zebrafish head and trunk in control, CdSe QDs and CdSe@PEG groups.n=20. Scale bars: $500 \mu \mathrm{m}$. 


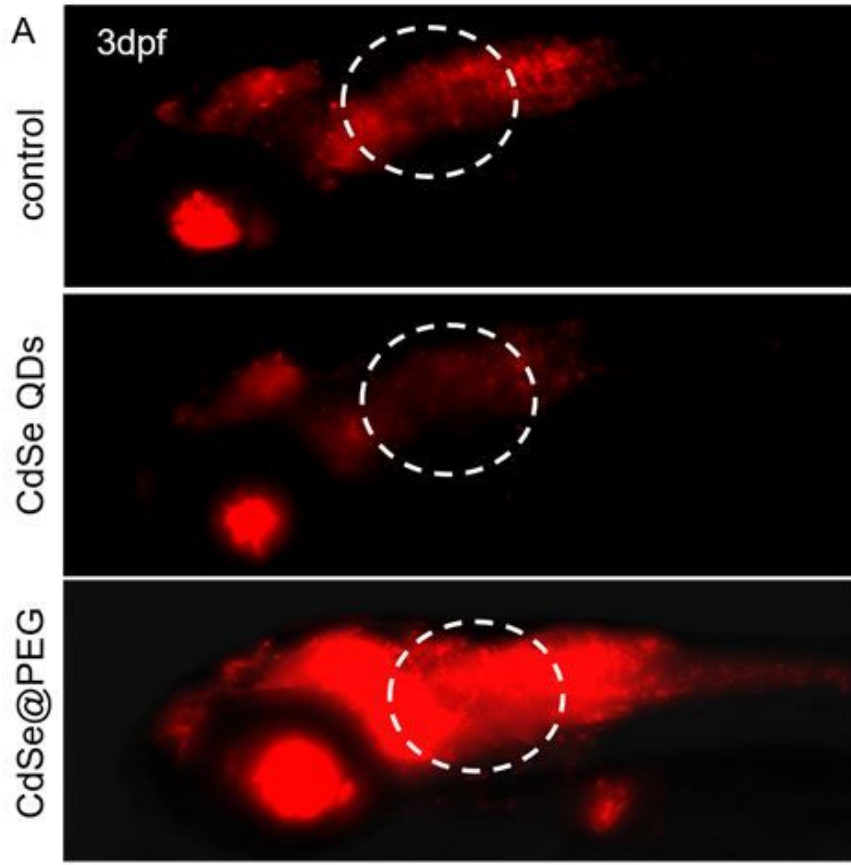

C

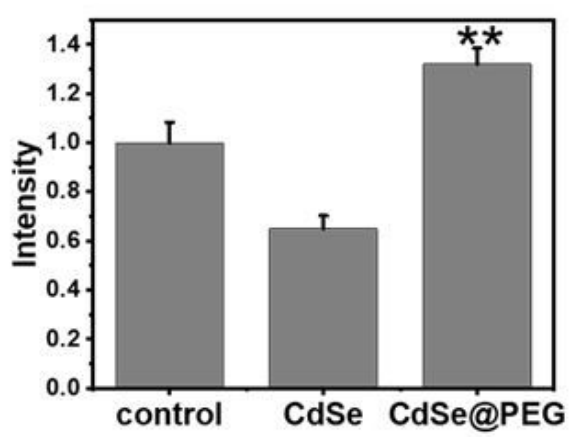

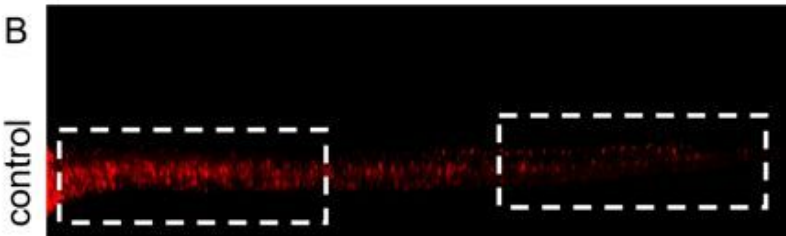
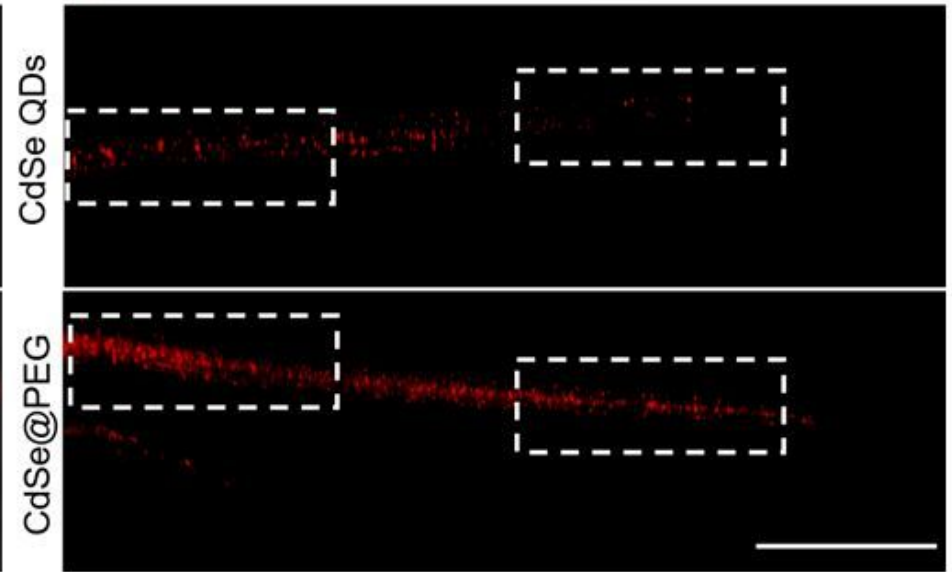

D

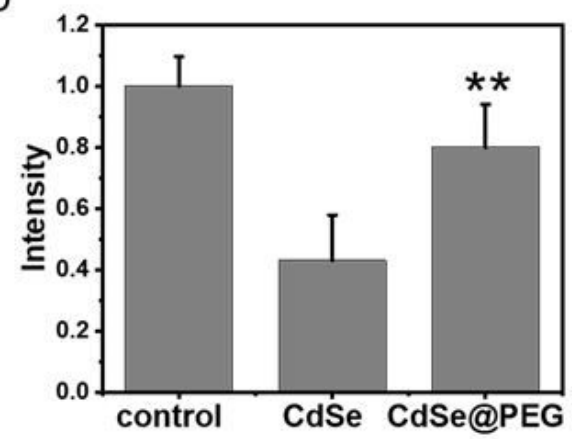

Figure 6

PEG-modified significantly decreased the nerve toxicity of CdSe QDs in zebrafish. (A, B) The effect of CdSe QDs, CdSe@PEG on neurons in zebrafish head and trunk. (C, D) Analysis of the intensity of neurons expression of zebrafish head and trunk in control, CdSe QDs and CdSe@PEG groups.n=20. Scale bars: $500 \mu \mathrm{m}$. 


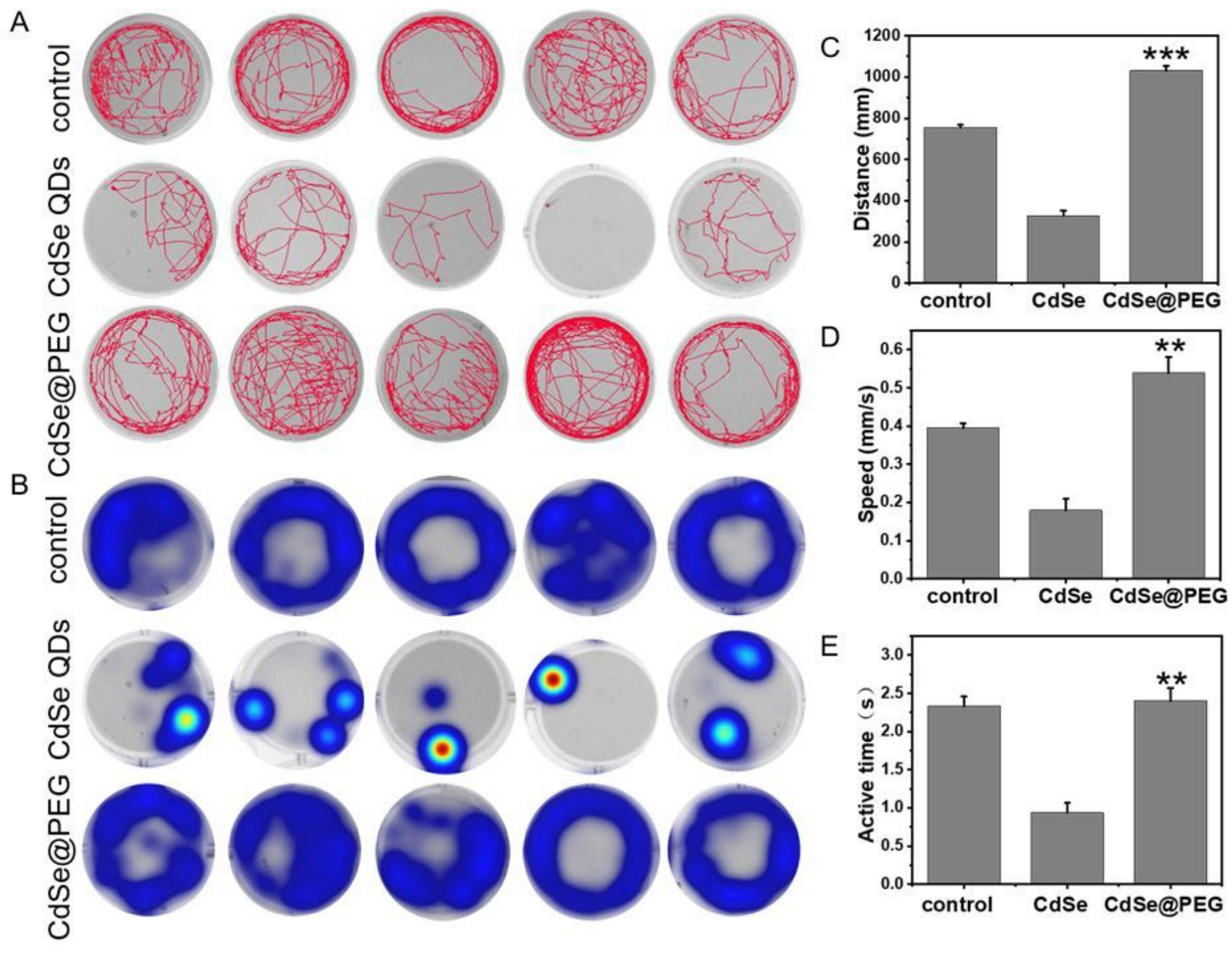

Figure 7

The effect of CdSe QDs and CdSe@PEG on zebrafish behavior. (A) Zebrafish behavior trajectories in the control, CdSe QDs and CdSe@PEG groups. (B) Zebrafish movement heat map of control, CdSe QDs and CdSe@PEG groups. (C, D, E) The total movement distance, movement speed and active time in the control, CdSe QDs and CdSe@PEG groups. n=20. 
A
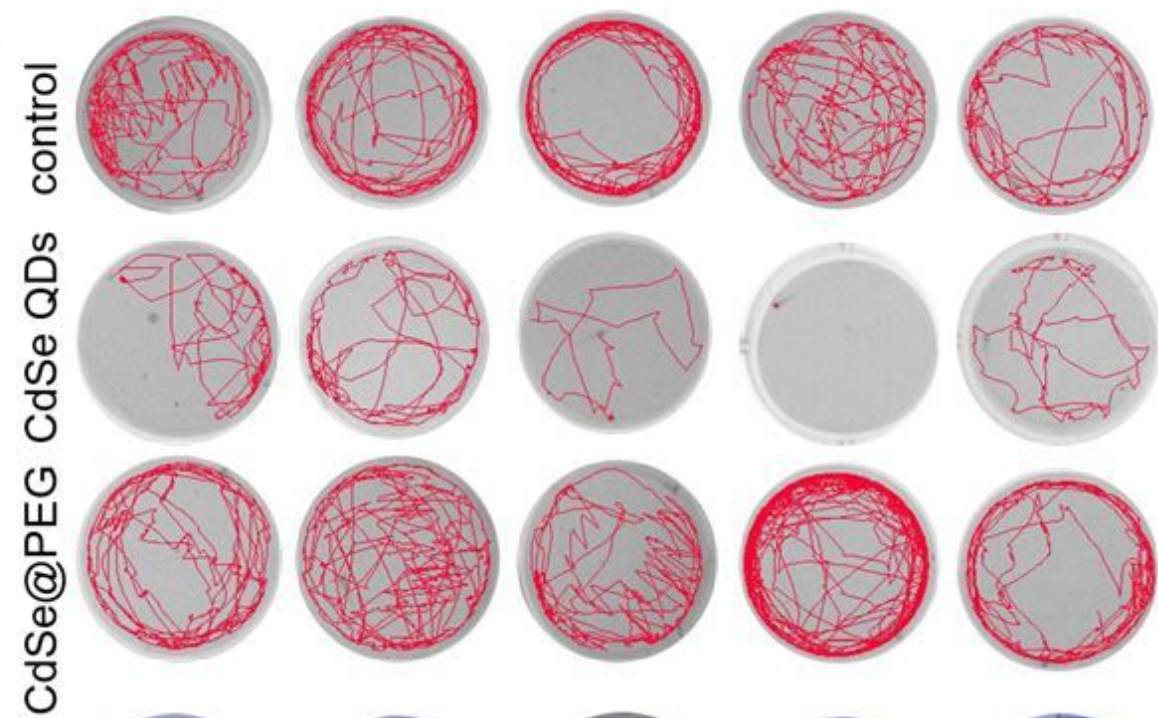

B
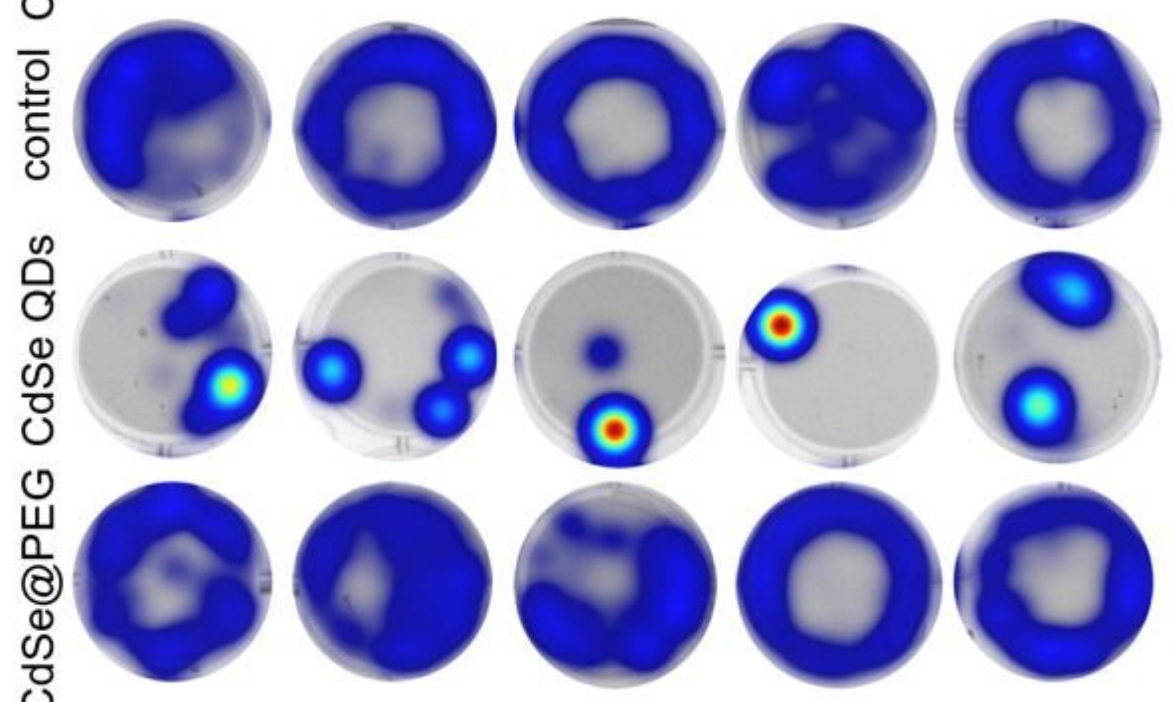

C

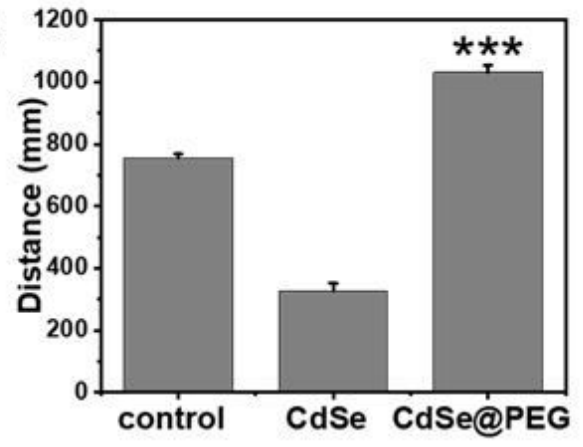

$\mathrm{D}$

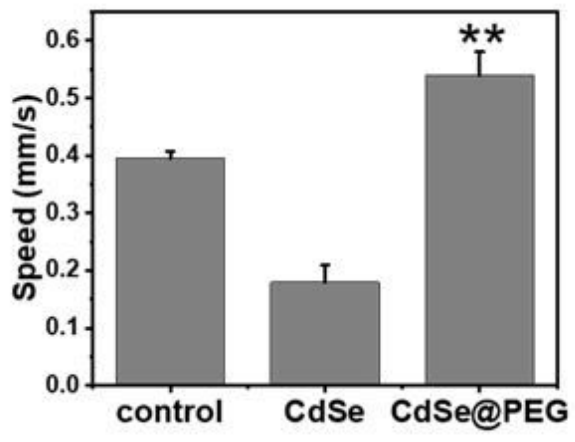

$\mathrm{E}$

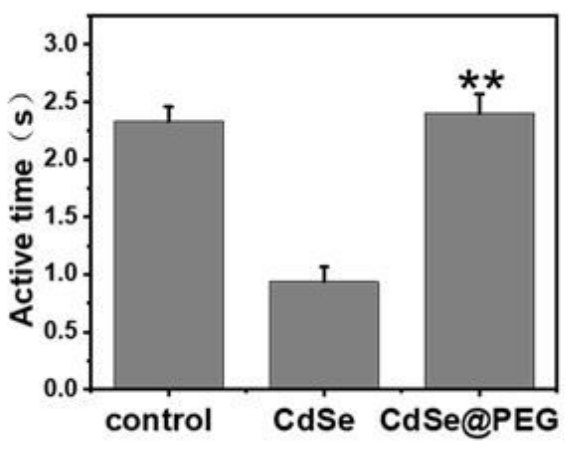

\section{Figure 7}

The effect of CdSe QDs and CdSe@PEG on zebrafish behavior. (A) Zebrafish behavior trajectories in the control, CdSe QDs and CdSe@PEG groups. (B) Zebrafish movement heat map of control, CdSe QDs and CdSe@PEG groups. (C, D, E) The total movement distance, movement speed and active time in the control, CdSe QDs and CdSe@PEG groups. n=20.

\section{Supplementary Files}

This is a list of supplementary files associated with this preprint. Click to download.

- Supportinginformation.doc

- Supportinginformation.doc

- TOCG.JPG

- TOCG.JPG 